\title{
CARACTERIZACIÓN Y RENDIMIENTO DE POBLACIONES DE MAÍZ NATIVAS DE MOLCAXAC, PUEBLA
}

\section{CHARACTERIZATION AND YIELD PERFORMANCE OF NATIVE MAIZE POPULATIONS FROM MOLCAXAC, PUEBLA}

\author{
Elizabeth Ángeles-Gaspar ${ }^{1}$, Enrique Ortiz-Torres ${ }^{1 *}$, Pedro A. López ${ }^{1}$ y Gustavo López-Romero²
}

\author{
${ }^{1}$ Postgrado en Estrategias para el Desarrollo Agrícola Regional, Colegio de Postgraduados-Campus Puebla. 72130, Puebla, Puebla. Tel. 01 (222)285-0738. \\ ${ }^{2}$ Colegio de Postgraduados-Campus Veracruz. 91700, Veracruz, Veracruz. \\ * Autor para correspondencia (enriqueortiz@colpos.mx)
}

\section{RESUMEN}

En Molcaxac, Puebla $98 \%$ de los productores de maíz (Zea mays L.) utilizan poblaciones nativas. El objetivo del estudio fue evaluar el rendimiento de grano y caracterizar agronómicamente una muestra de la diversidad de las poblaciones locales de maíz existente en este municipio. En 2007 se evaluaron 56 materiales genéticos -52 poblaciones locales y cuatro híbridos recomendados para la zonabajo temporal o secano, en tres localidades. Los resultados mostraron que existen poblaciones locales con mayor rendimiento de grano (MX-6, SA-2 y SC-3 con 5.6, 4.8 y $4.5 \mathrm{t} \mathrm{ha}^{-1}$ ) que las variedades introducidas, lo que muestra mejor adaptación de esas poblaciones nativas a las condiciones de suelo y clima de la región. El análisis de componentes principales definió que las variables con mayor influencia para explicar la variación observada fueron días a floración femenina, número de hojas arriba de la mazorca, área de la hoja de la mazorca, aspecto de planta, longitud de mazorca, número de hileras de grano por mazorca, diámetro de mazorca, longitud de grano, ancho de grano, grosor de grano, diámetro de olote y diámetro de médula. El análisis de conglomerados definió cinco grupos de poblaciones. Se concluye que en la región de Molcaxac, Puebla, existe variación amplia entre los materiales locales de maíz y algunos presentan buen rendimiento para las condiciones de temporal. La variación observada está parcialmente relacionada con el origen geográfico de las poblaciones dentro del municipio.

Palabras clave: Zea mays, diversidad genética, poblaciones locales, potencial productivo.

\section{SUMMARY}

In Molcaxac, Puebla $98 \%$ of maize (Zea mays L.) growers use local landrace populations. The aim of this study was to evaluate grain yield and agronomically characterize a sample of the local diversity maize populations from this country, Pue. Fifty-six genotypes $\mathbf{- 5 2}$ local populations and four hybrids recommended for that area- were evaluated in $\mathbf{2 0 0 7}$ under rainfed conditions, in three locations of the region. Results showed the existence of local populations with higher yield (MX-6, SA-2, and Sc-3 which yielded 5.6, 4.8 and $4.5 \mathrm{t} \mathrm{ha}^{-1}$ ) than the introduced ones, so these populations have a better adaptation to the prevailing regional soil and climate conditions. The principal component analysis defined that the most influential traits to explain the observed variation were: female flowering, number of leaves above the ear, area of ear leaf, plant appearance, ear length, number of kernel rows per ear, ear diameter, kernel length, kernel width, kernel thickness, cob diameter and pith diameter. The cluster analysis defined five groups of populations. It is concluded that there is a wide variation among local maize landraces in the Molcaxac, Pue. region, and that some landraces can produce a good yield under rainfed conditions. The observed variation is partly related to the geographical origin of the maize populations within the county.

Index words: Zea mays, local populations, genetic diversity, yield potential.

\section{INTRODUCCIÓN}

México es considerado como el centro de origen, domesticación y dispersión del maíz (Zea mays L.). A la fecha se han descrito 59 razas potencialmente diferentes (Ortega, 2003; Kato et al., 2009). Como en el continente americano se reportaron unas 300 razas, la variación en México representa $22.7 \%$ de la diversidad del maíz en el continente (Serratos, 2009). Esta diversidad está presente aun a nivel de microrregiones, a la que Muñoz (2005) denomina patrón etnofitogenético o patrón varietal y lo define como el conjunto de grupos de variedades de maíz, los estratos o niveles ambientales y las relaciones entre ellos.

Comúnmente cada grupo de maíz se diferencia de otro en precocidad, color de grano y usos. Adicionalmente, una variedad del grupo se siembra en un sitio particular del nicho o microrregión y en un periodo específico que depende de la humedad del suelo, la temperatura, la altitud o del inicio de las lluvias. El estrato o nivel ambiental es cada sitio del nicho con un régimen higrotérmico específico. El patrón varietal se puede 
describir con base en tres características básicas: color del grano, precocidad y otras características agronómicas, particular-mente rendimiento de grano (Gil et al., 2004). La diversidad de maíz se encuentra principalmente en donde imperan condiciones de temporal o secano y sistemas campesinos de producción (Herrera et al., 2000), y los agricultores generalmente disponen de más de una variedad nativa adaptada a su ambiente (Aceves et al., 2002).

En México el Estado de Puebla ocupa el octavo lugar en superficie cosechada de maíz (INEGI, 2010) y $93 \%$ de esa área se cultiva en condiciones de temporal (INEGI, 2007). Gil et al. (2004) estudiaron la diversidad de maíz en 15 microrregiones del Estado de Puebla y encontraron una amplia variación en días a floración femenina, color de grano y características agronómicas. Esta diversidad también ha sido reportada para el Valle de Puebla (Hortelano et al., 2008).

El maíz es el cultivo con mayor superficie en el municipio de Molcaxac, Puebla. Durante el periodo 20022003 se sembraron 3672 ha, de las cuales $74 \%$ se cultivaron en condiciones de temporal (INEGI, 2007), donde solamente se cosechó $35 \%$ de la producción total de grano. Los bajos rendimientos se deben principalmente a los suelos pobres y degradados y a la baja precipitación pluvial (650 $\mathrm{mm}$ anuales, en promedio) con un régimen de lluvias mal distribuido (Muñoz, 2005). Pese a estas adversidades, los productores cultivan maíz todos los años con fines de autoconsumo y como costumbre cultural. Esto ha permitido proteger y mantener la diversidad genética local. El aprovechamiento de las variedades locales puede ayudar a la preservación in situ de la diversidad genética local. Según Herrera et al. (2004), la conservación se facilita si se conoce la diversidad genética en las condiciones de la agricultura tradicional.

Por lo anterior, este trabajo tuvo como objetivo caracterizar la diversidad genética de una muestra representativa de las poblaciones locales de maíz existentes en el municipio de Molcaxac, Puebla, con base en variables agronómicas y morfológicas, así como evaluar su potencial de rendimiento.

\section{MATERIALES Y MÉTODOS}

Se evaluaron 52 poblaciones locales colectadas en troje (depósito rústico destinado a guardar la producción de maíz), con productores de seis localidades del municipio de Molcaxac, Puebla. Se obtuvieron 14 accesiones en Santa Cruz Huitziltepec, siete en San Andrés Mimihuapan, seis en San Luis Tehuizotla, ocho en Molcaxac, 15 en San José de Gracia y dos en Buenos
Aires; la Figura 1 muestra mazorcas de cuatro accesiones. Como variedades testigo se incluyeron el criollo del productor cooperante (TESPROD) y los híbridos comerciales 'Pantera' y 'H-7583' de la empresa Asgrow, y 'Piraña' y 'H-C-3311' de la empresa Caloro ADQ, TEC.

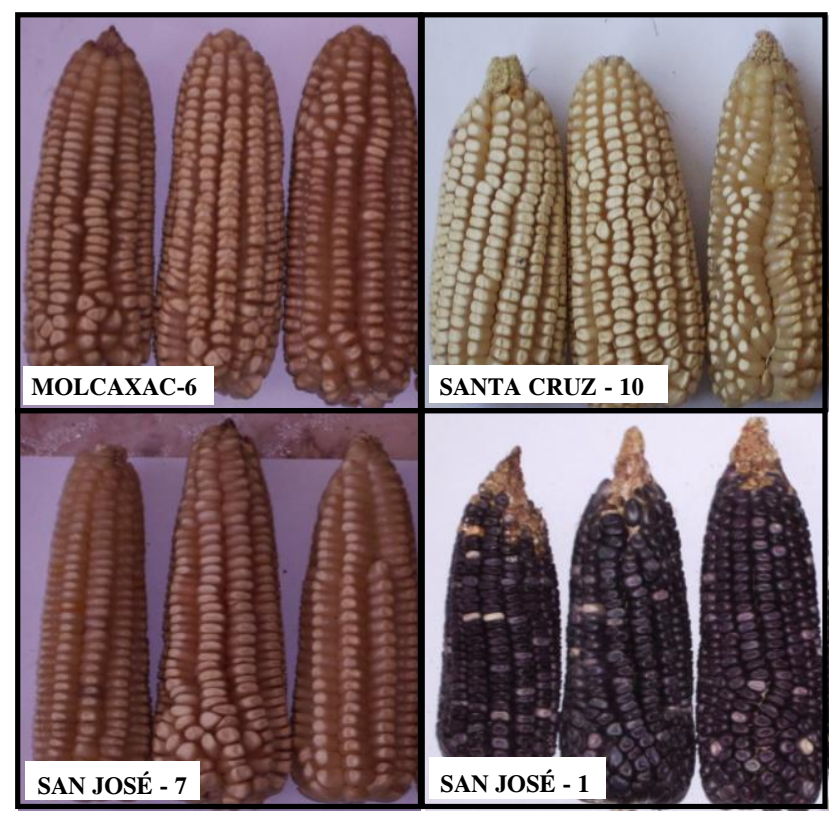

Figura 1. Mazorcas de cuatro accesiones de maíces nativos en Molcaxac, Puebla.

Los ensayos se establecieron en el municipio de Molcaxac, localizado en la parte centro sur del Estado de Puebla, en la región de la Mixteca Baja, a $18^{\circ} 44^{\prime} \mathrm{LN}$ y $97^{\circ}$ 54' LO, a una altitud de $1860 \mathrm{~m}$ (INEGI, 2004). Su clima es $\mathrm{BS}_{1}\left(\mathrm{~h}^{\prime}\right) \mathrm{w}(\mathrm{w}) \mathrm{ig}$, correspondiente a semiárido seco con lluvias principalmente en verano, con temperatura media anual sobre $22{ }^{\circ} \mathrm{C}$ y temperaturas menores a $18{ }^{\circ} \mathrm{C}$ en los meses más fríos. La precipitación media anual es de $650 \mathrm{~mm}$ (García, 1973). La evaluación se hizo en tres localidades, dos de las cuales (LOC1 y LOC2) se establecieron en el Rancho el Tecorral del Centro de Bachillerato Tecnológico Agropecuario No. 79 de Molcaxac. La localidad tres (LOC3) estuvo en la parcela de un agricultor cooperante de la comunidad de Molcaxac.

La siembra se hizo a "tapa pie", que es la forma tradicional en la región. Se depositaron tres semillas por punto para luego aclarear a dos plantas. La distancia entre plantas fue de $0.50 \mathrm{~m}$. La fertilización se hizo con la fórmula $180 \mathrm{~N}-80 \mathrm{P}-60 \mathrm{~K}$, la cual se dividió en dos aplicaciones: la mitad del $\mathrm{N}$ y todo el $\mathrm{P}$ y $\mathrm{K}$ en la primera labor, mientras que el resto del $\mathrm{N}$ se aplicó en la segunda labor. Los experimentos se condujeron en condiciones de 
temporal durante todo el ciclo de cultivo, de acuerdo con las prácticas culturales tradicionales de los productores.

El diseño experimental utilizado fue un látice rectangular 7 x 8 (Cochran y Cox, 1965), con dos repeticiones en cada localidad. La unidad experimental consistió en dos surcos de $5 \mathrm{~m}$ de largo, espaciados a 0.8 $\mathrm{m}$. Las variables evaluadas fueron divididas en cinco grupos:

1) Caracteres vegetativos, medidos en una muestra de cinco plantas por parcela: altura de planta (APLANT, en $\mathrm{cm})$, altura de mazorca (AMAZ, en $\mathrm{cm}$ ), número de hojas arriba de la mazorca (HOARMZ), número de hojas abajo de la mazorca (HOBMZ), largo de hoja de la mazorca (LHOJA, en $\mathrm{cm}$ ), ancho de hoja de la mazorca (ANHOJA, en $\mathrm{cm}$ ), diámetro de tallo (DTALLO, en $\mathrm{cm}$ ), total de hojas (THOJAS), índice de altura de mazorca/altura de planta (IAM/AP), y área de la hoja de la mazorca (AFOL, en $\mathrm{cm}^{2}$ ).

2) Caracteres agronómicos: rendimiento de grano (REN, en $\mathrm{kg} \mathrm{ha}^{-1}$ ), días a floración masculina (DFM) y femenina (DFF), asincronía floral (AF = DFF-DFM), cuateo (CUATEO = porcentaje de plantas por parcela con más de dos mazorcas), peso de olote (POLOTE, en gramos), calificación de mazorca (CALMAZ, con una escala de 1 a 5 , donde 1 correspondió a un buen aspecto de mazorca y 5 al peor), calificación de planta (CALPLA, con una escala de 1 a 5 , donde 1 correspondió a un buen aspecto de planta y 5 al peor), número de mazorcas por planta (NMZPP) y factor de desgrane (FACDES, que es el porcentaje del peso de la mazorca que corresponde al grano).

3) Caracteres de la espiga, medidos en una muestra de cinco plantas por parcela: longitud del pedúnculo (LPEDUN, en $\mathrm{cm}$ ), longitud del tramo ramificado (LTRAMI, en $\mathrm{cm}$ ), longitud de la rama central (LRCENT, en $\mathrm{cm}$ ), longitud de la espiga (LESPI, en $\mathrm{cm}$ ), y número total de ramas (NRAMAS).

4) Caracteres de la mazorca, medidos en una muestra de cinco mazorcas por parcela: longitud de mazorca (LMZ, en $\mathrm{cm}$ ); diámetros de mazorca (DMZ, en $\mathrm{cm})$, de olote $(\mathrm{DOLO}$, en $\mathrm{cm})$, de raquis (DRAQS, en $\mathrm{cm})$, de médula (DME, en $\mathrm{cm}$ ), como se ilustra en la Figura 2; número de hileras por mazorca (HILMZ) y número de granos por hilera (GRAHIL).

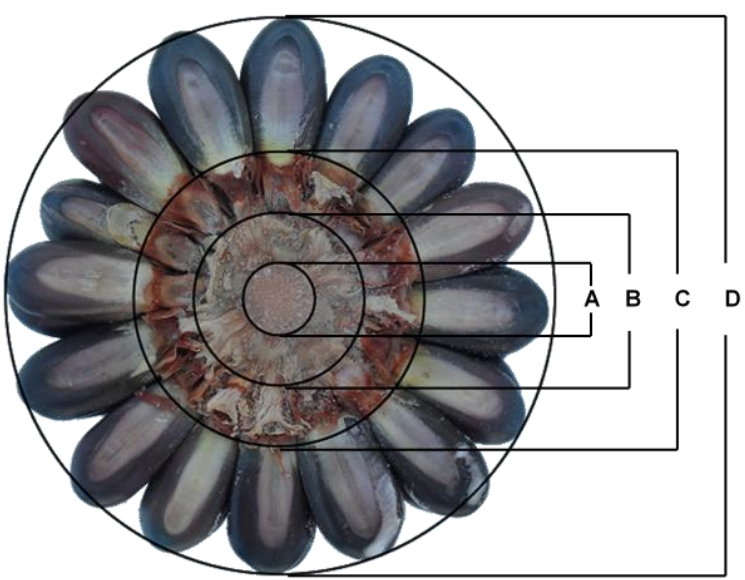

Figura 2. Variables de mazorca: A) Diámetro de médula; B) Diámetro de raquis; C) Diámetro de olote; D) Diámetro de mazorca.

5) Caracteres de grano, medidos en una muestra de 10 granos por parcela: longitud de grano (LGR, en $\mathrm{cm}$ ), ancho de grano (AGR, en $\mathrm{cm}$ ), grosor de grano (GGR, en $\mathrm{cm})$ y peso de 100 granos (P100GRA, en gramos) (IBPGR, 1991).

También se llevó un registro de las temperaturas extremas (máximas y mínimas) y de la precipitación durante el desarrollo del cultivo en cada localidad. Para obtener estos datos climáticos, cerca de los experimentos se instaló un pluviómetro de acumulación semanal y un termómetro de máximas y mínimas. Las lecturas de temperatura fueron tomadas diariamente, mientras que la precipitación pluvial se registró semanalmente.

Cada variable se sometió a un análisis de varianza combinado a través de localidades para determinar los efectos de la interacción genotipo $\mathrm{x}$ localidad y a una prueba de medias con la diferencia mínima significativa mediante el procedimiento GLM del programa SAS (SAS Institute, 2004). Posteriormente, se aplicó un análisis de componentes principales (ACP) a partir de la matriz de correlaciones entre los caracteres, con el procedimiento PRINCOMP de SAS. También se efectuó un análisis de conglomerados (ADC) con la matriz de distancias euclidianas; el método de agrupamiento aplicado fue el de mínima varianza dentro de grupos de Ward, y para mejorar la presentación de la grafica se aplicó el coeficiente de determinación como escala de disimilitud entre conglomerados (Mohammadi y Prasanna, 2003); este análisis se hizo con el procedimiento CLUSTER del programa SAS (SAS Institute, 2004).

\section{RESULTADOS Y DISCUSIÓN}

El análisis de varianza combinado reveló que hay diferencias significativas $(\mathrm{P} \leq 0.05)$ entre genotipos 
(GEN), en $75 \%$ de las 36 variables (Cuadro 1). Entre localidades (LOC) también se detectaron diferencias significativas $(\mathrm{P} \leq 0.05)$ en $94 \%$ de las variables, mientras que para la interacción genotipos $\mathrm{x}$ localidades (GEN x LOC) se presentaron diferencias significativas ( $\mathrm{P}$ $\leq 0.05)$ en $56 \%$ de las variables. El haber encontrado diferencias estadísticas entre genotipos y entre localidades, así como altos niveles de variación en los caracteres, indica la existencia de diversidad genética en las variedades evaluadas y que éstas responden de manera diferente en ambientes contrastantes. Lo anterior coincide con la magnitud de la diversidad encontrada en el Valle de Puebla por Hortelano et al. (2008). Sin embargo, 44 $\%$ de las variables no presentaron diferencias significativas en GEN x LOC, lo que significa que su expresión no es afectada de manera diferencial ante el cambio de genotipos a través de ambientes.

Cuadro 1. Cuadrados medios del análisis de varianza combinado y coeficientes de variación de las variables evaluadas en maíces nativos del municipio de Molcaxac, Pue. 2007.

\begin{tabular}{|c|c|c|c|c|c|}
\hline \multirow[t]{2}{*}{ Variable } & \multicolumn{4}{|c|}{ Cuadrados medios } & \multirow{2}{*}{$\begin{array}{l}\text { CV } \\
(\%)\end{array}$} \\
\hline & LOC & GEN & GEN X LOC & Error & \\
\hline \multicolumn{6}{|l|}{ I. Caracteres vegetativos } \\
\hline Altura de planta $(\mathrm{cm})$ & $12.27 * *$ & $0.062 \mathrm{~ns}$ & $0.041 \mathrm{~ns}$ & 0.0478 & 11.0 \\
\hline Altura de mazorca $(\mathrm{cm})$ & $3.73 * *$ & $0.058 * *$ & $0.035 \mathrm{~ns}$ & 0.030 & 14.2 \\
\hline Hojas arriba de la mazorca & $29.13 * *$ & $0.44 * *$ & $0.34 * *$ & 0.20 & 8.4 \\
\hline Hojas abajo de la mazorca & $233.75 * *$ & $1.78 *$ & $0.93 \mathrm{~ns}$ & 1.21 & 15.2 \\
\hline Ancho de hoja de la mazorca $(\mathrm{cm})$ & $8.60 * *$ & $0.90 * *$ & $0.68 * *$ & 0.42 & 6.9 \\
\hline Largo de hoja de la mazorca $(\mathrm{cm})$ & $4371.25 * *$ & $52.10 \mathrm{~ns}$ & $60.29 \mathrm{~ns}$ & 50.06 & 8.6 \\
\hline Total de hojas & $109.23 * *$ & $1.69 *$ & $0.78 \mathrm{~ns}$ & 166.53 & 8.3 \\
\hline Diámetro de tallo $(\mathrm{cm})$ & $0.046 \mathrm{~ns}$ & $0.039 * *$ & $0.027 \mathrm{~ns}$ & 0.023 & 7.2 \\
\hline Área de la hoja de la mazorca $\left(\mathrm{cm}^{2}\right)$ & $416496.76 * *$ & $10109.28 * *$ & $7527.90 * *$ & 4554.33 & 11.6 \\
\hline \multicolumn{5}{|l|}{ II. Caracteres agronómicos } & 9.2 \\
\hline Rendimiento (kg ha ${ }^{-1}$ ) & $315582157 * *$ & $1994316 * *$ & $1222423 \mathrm{~ns}$ & 1018209 & 29.3 \\
\hline Días a floración masculina & $114.15 * *$ & $29.30 * *$ & $18.53 *$ & 13.0 & 4.1 \\
\hline Días a floración femenina & $236.58 * *$ & $26.54 * *$ & $18.76 *$ & 12.92 & 4.01 \\
\hline Asincronía floral & $259.43 * *$ & $7.42 *$ & $7.03 *$ & 5.04 & 104.5 \\
\hline Cuateo & $0.67 \mathrm{~ns}$ & $7.39 \mathrm{~ns}$ & $9.09 *$ & 6.16 & 53.4 \\
\hline Peso de olote (g) & $9537.79 * *$ & $1055.02 * *$ & $1071.74 * *$ & 469.1 & 24.7 \\
\hline Calificación de mazorca & $4.54 * *$ & $0.22 * *$ & $0.25 * *$ & 0.121 & 10.2 \\
\hline Calificación de planta & $3.75 * *$ & $0.27 *$ & $0.32 * *$ & 0.175 & 12.2 \\
\hline Número de mazorcas por planta & $0.487 * *$ & $0.042 \mathrm{~ns}$ & $0.032 \mathrm{~ns}$ & 0.035 & 18.8 \\
\hline Factor de desgrane & $0.044 * *$ & $0.0019 * *$ & $0.0014 * *$ & 0.00064 & 3.0 \\
\hline \multicolumn{6}{|l|}{ III. Caracteres de la espiga } \\
\hline Número total de ramas & $19.78 *$ & $8.03 \mathrm{~ns}$ & $7.16 \mathrm{~ns}$ & 5.9 & 18.4 \\
\hline Longitud del pedúnculo $(\mathrm{cm})$ & $567.36 * *$ & $6.73 \mathrm{~ns}$ & $5.66 \mathrm{~ns}$ & 5.93 & 13.2 \\
\hline Longitud del tramo ramificado $(\mathrm{cm})$ & $80.03 * *$ & $2.88 \mathrm{~ns}$ & $3.06 \mathrm{~ns}$ & 2.57 & 15.3 \\
\hline Longitud de la rama central (cm) & $205.16 * *$ & $8.69 *$ & $6.73 \mathrm{~ns}$ & 5.46 & 9.5 \\
\hline Longitud de la espiga (cm) & $2115.37 * *$ & $14.44 \mathrm{~ns}$ & $12.87 \mathrm{~ns}$ & 14.43 & 7.1 \\
\hline \multicolumn{6}{|l|}{ IV. Caracteres de la mazorca } \\
\hline Longitud de la mazorca (cm) & $14.86 * *$ & $3.64 * *$ & $3.23 * *$ & 1.81 & 10.2 \\
\hline Hileras por mazorca & $8.92 *$ & $10.77 * *$ & $3.85 *$ & 2.59 & 12.2 \\
\hline Granos por hilera & $123.52 * *$ & $11.85 \mathrm{~ns}$ & $17.0 *$ & 11.76 & 12.5 \\
\hline Diámetro de la mazorca $(\mathrm{cm})$ & $14.64 * *$ & $0.17 * *$ & $0.13 \mathrm{~ns}$ & 0.098 & 7.2 \\
\hline Diámetro de olote $(\mathrm{cm})$ & $0.87 * *$ & $0.12 * *$ & $0.068 * *$ & 0.04 & 9.3 \\
\hline Diámetro de raquis $(\mathrm{cm})$ & $0.83 * *$ & $0.058 * *$ & $0.038 * *$ & 0.023 & 12.1 \\
\hline Diámetro de médula (cm) & $0.236 * *$ & $0.027 * *$ & $0.026 * *$ & 0.016 & 23.6 \\
\hline \multicolumn{6}{|l|}{ V. Caracteres de grano } \\
\hline Longitud de grano $(\mathrm{cm})$ & $146.76 * *$ & $3.94 * *$ & $2.40 *$ & 1.76 & 9.8 \\
\hline Ancho de grano (cm) & $6.22 * *$ & $1.48 * *$ & $0.83 * *$ & 0.40 & 7.5 \\
\hline Grosor de grano $(\mathrm{cm})$ & $1.43 * *$ & $0.43 * *$ & $0.28 \mathrm{~ns}$ & 0.25 & 13.1 \\
\hline Peso de 100 granos $(\mathrm{g})$ & $3359.39 * *$ & $65.46 * *$ & $55.93 * *$ & 28.35 & 16.3 \\
\hline
\end{tabular}

$*=\mathrm{P} \leq 0.05, * *=\mathrm{P} \leq 0.01 ; \mathrm{ns}=$ no significativa; GEN x LOC = genotipo x localidad; CV = coeficiente de variación; GEN = genotipo; LOC = localidad. Cuateo $=$ porcentaje de plantas por parcela con más de dos mazorcas; Calificación de mazorca = con una escala de uno a cinco, donde uno correspondió a un buen aspecto de mazorca y cinco al peor; Calificación de planta = con una escala de uno a cinco, donde uno correspondió a un buen aspecto de planta y cinco al peor; Factor de desgrane = porcentaje del peso de la mazorca que corresponde al grano; Diámetro de médula $=$ distancia $(\mathrm{cm})$ a través del olote entre los puntos extremos que delimitan el tejido esponjoso y el tejido endurecido del raquis. 
Las diferencias estadísticas $(\mathrm{P} \leq 0.05)$ entre genotipos indican diferentes potenciales productivos entre ellos, que no fueron afectados por la interacción GEN x LOC. Al respecto, hubo poblaciones nativas que superaron o igualaron en rendimiento de grano a las variedades comerciales recomendadas para la región (Cuadro 2). Tres poblaciones locales (MX-6, SA-2 y SC-3) se ubicaron estadísticamente en el grupo superior de rendimiento con 5.6, 4.8, $4.5 \mathrm{t} \mathrm{ha}^{-1}$, respectivamente. $\mathrm{La}$ población local MX-6 superó en $24 \%$ al mejor maíz mejorado ('H-C-3311'), lo que refleja la mejor adaptación de estos genotipos a las condiciones ambientales particulares de Molcaxac, Pue. Según Cervantes y Mejía (1984), Estrada et al. (1987) y Castillo et al. (1987), las poblaciones nativas del Valle de Puebla, de la región de Chiautla, Pue. y de la Mixteca Guerrerense son tan sobresalientes como las variedades comerciales recomendadas para esas regiones.

El mejor desempeño y adaptabilidad de las poblaciones nativas con respecto a las variedades mejoradas introducidas se atribuye a que las variedades mejoradas y los híbridos manifiestan su potencial productivo generalmente en condiciones óptimas de fertilización, humedad, manejo agronómico, control de plagas y enfermedades, similares al ambiente donde fueron obtenidas en campos experimentales (Centro de Investigaciones Agrarias, 1980). Es decir, existen variedades nativas que poseen mejores características en términos de comportamiento agronómico y adaptación al ambiente, debido a su amplia variación genética intrapoblacional y a su buen comportamiento per se, ventajas que pueden aprovecharse con fines de mejoramiento genético para áreas de temporal deficiente (Gil et al., 2004). Es posible que las variedades mejoradas no hayan mostrado su potencial de rendimiento porque su ciclo de crecimiento ocurrió en condiciones ambientales adversas, pues sólo se registraron $122 \mathrm{~mm}$ de lluvia en toda la estación de crecimiento. Lo anterior puede explicarse con lo señalado por Barrales et al. (1984), quienes afirmaron que el rendimiento está asociado con la cantidad de agua disponible durante el periodo de floración femenina, etapa muy sensible al déficit de humedad.

Al comparar el inicio de la floración entre localidades se observó que la floración femenina se adelantó ligeramente en la LOC3 donde inició a los 81 d después de la siembra (dds), mientras que en LOC1 y LOC2 inició a los 83 dds. La floración femenina promedio fue de 99 , 101 y 94 d en LOC1, LOC2 y LOC3, respectivamente. Es posible entonces inferir que algunas poblaciones nativas de esta zona presentaron un mejor comportamiento agronómico que las variedades o hibridos comerciales, debido a que las poblaciones se establecieron en las condiciones termopluviométricas y agroecológicas particulares en las cuales han evolucionado. De acuerdo con Barrales et al. (1984), las variedades precoces generalmente alcanzan evadir los periodos de deficiencias de agua que se manifiestan cuando hay baja precipitación pluvial.

Las poblaciones locales sobresalientes en rendimiento fueron más precoces que las variedades mejoradas ' $\mathrm{H}-\mathrm{C}$ 3311' y 'Pantera'. En el Cuadro 2 se muestra que hay diferencias $(\mathrm{P} \leq 0.05)$ en días a floración femenina entre las tres variedades de mayor rendimiento. La población SA2 tuvo $86.3 \mathrm{~d}$ y las MX6 y SC3 $90 \mathrm{~d}$, mientras que ' $\mathrm{H}$ C-3311' y 'Pantera' tuvieron 92.7 y $93.2 \mathrm{~d}$, lo que tal vez permitió a las variedades nativas un mejor aprovechamiento del agua de lluvia; en cambio, los materiales tardíos no dispusieron de suficiente humedad para completar su desarrollo, y como consecuencia presentaron menores rendimientos de grano. Se demuestra así la mejor adaptación de las poblaciones locales a las condiciones que prevalecen en esta región de la Mixteca Baja. Según López et al. (2005), existe una estrecha correspondencia entre la diversidad genética local y la variación ecológica ambiental, debido a que las plantas tardías se siembran en zonas con mayor humedad y con menor evaporación, mientras que las de ciclo corto se cultivan en áreas de menos lluvia y más evaporación. Sin embargo, esto no aplica para las variedades 'Piraña' y 'H7583', porque fueron de igual precocidad que las variedades nativas de mayor rendimiento.

La precocidad es una variable importante para denotar la adaptación de las poblaciones a las condiciones de humedad imperantes (Gil et al., 2004). La variable DFF mostró diferencias $(\mathrm{P} \leq 0.05)$ en las tres fuentes de variación (GEN, LOC y GEN x LOC). La variación en días a floración femenina se dividió en tres grupos para clasificar a las poblaciones: en precoces (84 a $87 \mathrm{~d}$ ), intermedias ( 88 a 91 d) y tardías ( 92 a 95 d). La floración femenina de los 56 maíces evaluados se comportó de la siguiente manera: 17 variedades fueron precoces (30\%), 29 intermedias (52\%) y 10 tardías (18 \%). La mayor frecuencia de poblaciones precoces e intermedias $(82.3$ $\%$ ) puede deberse a la existencia de una corta estación de crecimiento, ya que las lluvias se establecen generalmente en junio y terminan en octubre. 
Cuadro 2. Promedios de algunas variables morfológicas significativas en las 23 accesiones con mayor rendimiento de grano y en los testigos evaluados en tres localidades del municipio de Molcaxac, Pue. en 2007.

\begin{tabular}{|c|c|c|c|c|c|c|c|c|c|c|c|c|}
\hline Genotipo & $\begin{array}{c}\text { REN } \\
\left(\mathrm{kg} \mathrm{ha}^{-1}\right)\end{array}$ & DFF & $\begin{array}{c}\text { AMAZ } \\
(\mathrm{cm})\end{array}$ & HOARMZ & $\begin{array}{l}\text { AFOL } \\
\left(\mathrm{cm}^{2}\right)\end{array}$ & $\begin{array}{c}\text { P100GRA } \\
(\mathrm{g})\end{array}$ & $\begin{array}{l}\mathrm{LMZ} \\
(\mathrm{cm})\end{array}$ & $\begin{array}{c}\mathrm{DMZ} \\
(\mathrm{cm})\end{array}$ & $\begin{array}{l}\text { LGR } \\
(\mathrm{cm})\end{array}$ & $\begin{array}{l}\text { AGR } \\
(\mathrm{cm})\end{array}$ & $\begin{array}{l}\text { GGR } \\
(\mathrm{cm})\end{array}$ & FACDES \\
\hline MX6 & 5607 a & $90.0 \mathrm{~b}$ & $1.39 \mathrm{c}$ & $5.13 \mathrm{c}$ & $625.3 \mathrm{~b}$ & $37.8 \mathrm{a}$ & $13.8 \mathrm{a}$ & $4.45 \mathrm{a}$ & $13.4 \mathrm{~b}$ & $9.41 \mathrm{a}$ & $4.07 \mathrm{a}$ & $0.85 \mathrm{~b}$ \\
\hline SA2 & $4791 \mathrm{a}$ & $86.3 \mathrm{a}$ & $1.14 \mathrm{~b}$ & $5.57 \mathrm{c}$ & $641.1 \mathrm{~b}$ & $30.1 \mathrm{~b}$ & $12.8 \mathrm{~b}$ & $4.18 \mathrm{~b}$ & $13.5 \mathrm{a}$ & $7.84 \mathrm{c}$ & $3.83 \mathrm{~b}$ & $0.85 \mathrm{~b}$ \\
\hline $\mathrm{SC} 3$ & $4502 \mathrm{a}$ & $90.0 \mathrm{~b}$ & $1.29 \mathrm{~b}$ & $5.23 \mathrm{c}$ & $557.6 \mathrm{c}$ & $32.5 \mathrm{~b}$ & $13.1 \mathrm{~b}$ & $4.67 \mathrm{a}$ & $13.8 \mathrm{a}$ & $7.91 \mathrm{c}$ & $3.67 \mathrm{~b}$ & $0.86 \mathrm{a}$ \\
\hline SA6 & $4323 \mathrm{~b}$ & $87.8 \mathrm{~b}$ & $1.18 \mathrm{~b}$ & $5.57 \mathrm{c}$ & $599.0 \mathrm{~b}$ & $36.8 \mathrm{a}$ & $12.7 \mathrm{~b}$ & $4.52 \mathrm{a}$ & $14.0 \mathrm{a}$ & $9.23 \mathrm{a}$ & $3.70 \mathrm{~b}$ & $0.87 \mathrm{a}$ \\
\hline 'H-C-3311' & $4260 \mathrm{~b}$ & $92.7 \mathrm{c}$ & $1.02 \mathrm{a}$ & $6.60 \mathrm{a}$ & $749.4 \mathrm{a}$ & $28.0 \mathrm{~b}$ & $15.2 \mathrm{a}$ & $4.29 \mathrm{~b}$ & $11.6 \mathrm{c}$ & $8.37 \mathrm{~b}$ & $3.83 \mathrm{~b}$ & $0.82 \mathrm{~b}$ \\
\hline SJ7 & $4209 \mathrm{~b}$ & $85.7 \mathrm{a}$ & $1.25 \mathrm{~b}$ & $5.47 \mathrm{c}$ & $592.1 \mathrm{~b}$ & $34.9 \mathrm{a}$ & $14.2 \mathrm{a}$ & $4.09 \mathrm{~b}$ & $13.5 \mathrm{a}$ & $8.99 \mathrm{~b}$ & $4.20 \mathrm{a}$ & $0.86 \mathrm{a}$ \\
\hline SA7 & $4130 \mathrm{~b}$ & $85.3 \mathrm{a}$ & $1.17 \mathrm{~b}$ & $5.23 \mathrm{c}$ & $647.1 \mathrm{~b}$ & $39.5 \mathrm{a}$ & $14.6 \mathrm{a}$ & $4.44 \mathrm{a}$ & $14.0 \mathrm{a}$ & $9.33 \mathrm{a}$ & $3.87 \mathrm{a}$ & $0.88 \mathrm{a}$ \\
\hline SC5 & $3951 \mathrm{~b}$ & $89.7 \mathrm{~b}$ & $1.38 \mathrm{c}$ & $5.17 \mathrm{c}$ & $599.5 \mathrm{~b}$ & $34.8 \mathrm{a}$ & $14.1 \mathrm{a}$ & $4.58 \mathrm{a}$ & $13.6 \mathrm{a}$ & $8.84 \mathrm{~b}$ & $3.93 \mathrm{a}$ & $0.86 \mathrm{a}$ \\
\hline MX1 & $3935 \mathrm{~b}$ & $92.8 \mathrm{c}$ & $1.30 \mathrm{~b}$ & $5.60 \mathrm{~b}$ & $626.9 \mathrm{~b}$ & $33.9 \mathrm{~b}$ & $14.0 \mathrm{a}$ & $4.58 \mathrm{a}$ & $12.9 \mathrm{~b}$ & $8.65 \mathrm{~b}$ & $3.60 \mathrm{~b}$ & $0.83 \mathrm{~b}$ \\
\hline 'Piraña' & $3921 \mathrm{~b}$ & $90.3 \mathrm{~b}$ & $0.98 \mathrm{a}$ & $6.67 \mathrm{a}$ & $782.0 \mathrm{a}$ & $28.0 \mathrm{~b}$ & $15.2 \mathrm{a}$ & $4.20 \mathrm{~b}$ & $11.4 \mathrm{c}$ & $8.71 \mathrm{~b}$ & $3.63 \mathrm{~b}$ & $0.78 \mathrm{c}$ \\
\hline SL5 & $3890 \mathrm{~b}$ & $85.7 \mathrm{a}$ & $1.24 \mathrm{~b}$ & $5.17 \mathrm{c}$ & $576.2 \mathrm{~b}$ & $29.0 \mathrm{~b}$ & $13.9 \mathrm{a}$ & $4.56 \mathrm{a}$ & $14.4 \mathrm{a}$ & $8.28 \mathrm{c}$ & $3.53 \mathrm{~b}$ & $0.88 \mathrm{a}$ \\
\hline TESPROD & $3872 \mathrm{~b}$ & $91.0 \mathrm{~b}$ & $1.33 \mathrm{~b}$ & $5.40 \mathrm{c}$ & $594.1 \mathrm{~b}$ & $29.9 \mathrm{~b}$ & $12.8 \mathrm{~b}$ & $4.31 \mathrm{~b}$ & $13.3 \mathrm{~b}$ & $8.51 \mathrm{~b}$ & $3.53 \mathrm{~b}$ & $0.86 \mathrm{a}$ \\
\hline SJ1 & $3821 \mathrm{~b}$ & $87.0 \mathrm{a}$ & $1.27 \mathrm{~b}$ & $5.23 \mathrm{c}$ & $603.5 \mathrm{~b}$ & $33.7 \mathrm{~b}$ & $12.7 \mathrm{~b}$ & $4.43 \mathrm{a}$ & $13.7 \mathrm{a}$ & $8.20 \mathrm{c}$ & $4.00 \mathrm{a}$ & $0.88 \mathrm{a}$ \\
\hline SL2 & $3722 \mathrm{~b}$ & $87.3 \mathrm{a}$ & $1.27 \mathrm{~b}$ & $5.53 \mathrm{c}$ & $613.1 \mathrm{~b}$ & $35.8 \mathrm{a}$ & $13.1 \mathrm{~b}$ & $4.38 \mathrm{~b}$ & $14.0 \mathrm{a}$ & $8.67 \mathrm{~b}$ & $3.87 \mathrm{a}$ & $0.87 \mathrm{a}$ \\
\hline SL6 & 3692 b & $85.7 \mathrm{a}$ & $1.21 \mathrm{~b}$ & $5.17 \mathrm{c}$ & $527.3 \mathrm{c}$ & $31.5 \mathrm{~b}$ & $12.7 \mathrm{~b}$ & $4.31 \mathrm{~b}$ & $14.1 \mathrm{a}$ & $8.10 \mathrm{c}$ & $3.53 \mathrm{~b}$ & $0.89 \mathrm{a}$ \\
\hline $\mathrm{SA} 3$ & $3672 \mathrm{~b}$ & $86.0 \mathrm{a}$ & $1.16 \mathrm{~b}$ & $5.13 \mathrm{c}$ & $621.4 \mathrm{~b}$ & $32.0 \mathrm{~b}$ & $12.4 \mathrm{~b}$ & $4.76 \mathrm{a}$ & $14.7 \mathrm{a}$ & $7.99 \mathrm{c}$ & $3.70 \mathrm{~b}$ & $0.88 \mathrm{a}$ \\
\hline $\mathrm{SJ} 3$ & $3666 \mathrm{~b}$ & $86.7 \mathrm{a}$ & $1.18 \mathrm{~b}$ & $5.17 \mathrm{c}$ & $590.6 \mathrm{~b}$ & $40.4 \mathrm{a}$ & $14.0 \mathrm{a}$ & $4.10 \mathrm{~b}$ & $12.8 \mathrm{~b}$ & $9.77 \mathrm{a}$ & $4.43 \mathrm{a}$ & $0.86 \mathrm{a}$ \\
\hline SJ12 & $3659 \mathrm{~b}$ & $86.5 \mathrm{a}$ & $1.21 \mathrm{~b}$ & $5.07 \mathrm{c}$ & $540.7 \mathrm{c}$ & $34.5 \mathrm{a}$ & $13.2 \mathrm{~b}$ & $4.26 \mathrm{~b}$ & $13.5 \mathrm{a}$ & $8.82 \mathrm{~b}$ & $3.47 \mathrm{~b}$ & $0.87 \mathrm{a}$ \\
\hline SJ11 & $3618 \mathrm{~b}$ & $88.0 \mathrm{~b}$ & $1.25 \mathrm{~b}$ & $5.17 \mathrm{c}$ & $583.9 \mathrm{~b}$ & $34.0 \mathrm{~b}$ & $13.2 \mathrm{~b}$ & $4.46 \mathrm{a}$ & $15.0 \mathrm{a}$ & $8.60 \mathrm{~b}$ & $3.53 \mathrm{~b}$ & $0.88 \mathrm{a}$ \\
\hline SA4 & $3614 \mathrm{~b}$ & $83.7 \mathrm{a}$ & $1.05 \mathrm{a}$ & $5.00 \mathrm{~d}$ & $527.6 \mathrm{c}$ & $31.4 \mathrm{~b}$ & $12.8 \mathrm{~b}$ & $4.44 \mathrm{a}$ & $14.5 \mathrm{a}$ & $8.16 \mathrm{c}$ & $3.52 \mathrm{~b}$ & $0.89 \mathrm{a}$ \\
\hline SJ5 & $3571 \mathrm{~b}$ & $88.2 \mathrm{~b}$ & $1.18 \mathrm{~b}$ & $5.00 \mathrm{~d}$ & $596.9 \mathrm{~b}$ & $37.2 \mathrm{a}$ & $13.0 \mathrm{~b}$ & $4.29 \mathrm{~b}$ & $13.5 \mathrm{a}$ & $8.67 \mathrm{~b}$ & $4.10 \mathrm{a}$ & $0.87 \mathrm{a}$ \\
\hline 'Pantera' & $3094 \mathrm{c}$ & $93.2 \mathrm{c}$ & $0.87 \mathrm{a}$ & $6.10 \mathrm{~b}$ & $592.9 \mathrm{~b}$ & $24.9 \mathrm{c}$ & $13.7 \mathrm{a}$ & $4.10 \mathrm{~b}$ & $11.6 \mathrm{c}$ & $8.10 \mathrm{c}$ & $3.90 \mathrm{a}$ & $0.81 \mathrm{c}$ \\
\hline 'H-7583' & $2448 \mathrm{c}$ & $89.2 \mathrm{~b}$ & $0.99 \mathrm{a}$ & $5.27 \mathrm{c}$ & $598.5 \mathrm{~b}$ & $33.3 \mathrm{~b}$ & $14.2 \mathrm{a}$ & $4.26 \mathrm{~b}$ & $13.1 \mathrm{~b}$ & $8.76 \mathrm{~b}$ & $3.87 \mathrm{a}$ & $0.84 \mathrm{~b}$ \\
\hline DMS & 1151 & 4.1 & 0.19 & 0.51 & 77 & 6.1 & 1.53 & 0.35 & 1.5 & 0.72 & 0.57 & 0.03 \\
\hline
\end{tabular}

Medias con la misma letra en columnas son iguales estadísticamente $(\mathrm{P}<0.05)$. REN = rendimiento; DFF = días a floración femenina; AMAZ = altura de mazorca; HOARMZ = número de hojas arriba de la mazorca; AFOL = área de la hoja de mazorca; P100GRA = peso de cien granos; LMZ = longitud de mazorca; DMZ = diámetro de mazorca; LGR = longitud de grano; AGR $=$ ancho de grano; GGR $=$ grosor de grano; FACDES $=$ factor de desgrane $=$ peso de grano/(peso de olote + peso de grano); TESPROD $=$ testigo del producto 
Gil et al. (2004) reportaron la diversidad de maíz en el Estado de Puebla, por el número de variedades identificadas, su precocidad y por la variabilidad de colores de grano. En este estudio se encontró que $84 \%$ de las poblaciones nativas presentó grano de color blanco, $14 \%$ azul y $2 \%$ amarillo. Al considerar el rendimiento por grupo de color, se registraron $3.6 \mathrm{t} \mathrm{ha}^{-1}$ en las poblaciones con grano amarillo y $3.4 \mathrm{t} \mathrm{ha}^{-1}$ en las variedades de grano blanco y azul, sin diferencias significativas entre estas últimas.

Según López y Muñoz (1984), en los Valles Altos de México el campesino ha establecido de forma empírica una escala de precocidad asociada con el color del grano, y agregaron que las poblaciones de grano amarillo fueron las más rendidoras; sin embargo, entre las poblaciones de maíz blanco hubo todos los niveles de precocidad, aunque fueron más frecuentes las de tipo intermedio con $47 \%$ del grupo, mientras que las poblaciones de grano blanco de tipo precoz y tardío representaron 31 y $21 \%$, respectivamente. De las variedades con grano azul, siete fueron intermedias $(88 \%)$ y una precoz $(12 \%)$. En nuestro estudio, la variedad amarilla fue de tipo precoz, lo que coincide con lo encontrado por López y Muñoz (1984).

La diversidad en color de grano y en precocidad es común entre las poblaciones nativas. Al respecto, Gil et al. (2004) señalaron que existe variabilidad genética entre los materiales locales y entre nichos ecológicos, en términos de color del grano, nivel de precocidad y características agronómicas, variabilidad que es atribuible a la selección efectuada durante generaciones por los productores, para tipo y color de grano -según los usos-. Hortelano et al. (2008) también señalaron que en el Valle de Puebla la variación genética existente puede agruparse de acuerdo con el color del grano.

El análisis de componentes principales mostró que los primeros tres componentes en conjunto explicaron $57.8 \%$ de la variación fenotípica observada en las poblaciones aquí estudiadas, con valores propios de $4.9,3.9$ y 2.1 , y contribución especifica de 25.9, 20.8 y $11 \%$ de la variabilidad total, respectivamente. De acuerdo con los vectores propios, en el primer componente las variables originales con mayor peso fueron: número de hojas arriba de la mazorca, área foliar de la hoja de la mazorca, calificación de planta, diámetro de médula y diámetro de olote. En el segundo componente, las variables originales de mayor importancia fueron: longitud de la mazorca, número de hileras por mazorca, diámetro de mazorca y ancho de grano. El tercer componente estuvo fuertemente influido por las variables: días a floración femenina, longitud de grano y grosor de grano (Cuadro 3).
Cuadro 3. Vectores propios para los tres primeros componentes principales y 19 variables originales medidas en la caracterización de 56 variedades de maíz, en el municipio de Molcaxac, Pue. 2007

\begin{tabular}{lccc}
\hline Variable & CP1 & CP2 & CP3 \\
\hline DFF & 0.2368 & 0.1662 & $\mathbf{0 . 4 2 5 0}$ \\
AF & -0.0693 & 0.1572 & 0.1354 \\
IAM_AP & -0.1944 & 0.1879 & 0.2534 \\
HAMZ & $\mathbf{0 . 3 6 6 1 *}$ & -0.1024 & -0.1273 \\
AFOL & $\mathbf{0 . 3 3 6 5}^{*}$ & -0.1042 & -0.1972 \\
THOJAS & 0.1872 & 0.2275 & 0.2650 \\
DTALLO & 0.2330 & 0.0548 & -0.0171 \\
NRAMA & -0.0979 & 0.2581 & 0.1155 \\
LRCENT & 0.2639 & -0.0675 & -0.2465 \\
CALMAZ & -0.1561 & 0.0978 & 0.2349 \\
CALPLA & $-\mathbf{0 . 3 3 9 0} *$ & -0.0807 & -0.0504 \\
LMZ & 0.1809 & $-\mathbf{0 . 3 5 5} *$ & -0.1009 \\
HILMZ & 0.1211 & $\mathbf{0 . 4 2 6 9 *}$ & -0.0761 \\
DMZ & 0.0240 & $\mathbf{0 . 3 5 5 5}$ & -0.2111 \\
LGR & -0.2461 & 0.2482 & $-\mathbf{0 . 3 6 7 1} *$ \\
AGR & -0.0345 & $\mathbf{- 0 . 3 9 1 5 *}$ & 0.1715 \\
GGR & -0.0030 & -0.2249 & $\mathbf{0 . 4 7 9 0} *$ \\
DOLO & $\mathbf{0 . 3 3 5 8 *}$ & 0.1473 & 0.1458 \\
DME & $\mathbf{0 . 3 5 1 8 *}$ & 0.1640 & 0.0621 \\
\hline
\end{tabular}

* = Variable que explica mayor variación; DFF $=$ dias a floración femenina; $\mathrm{AF}=$ asincrónica floral; $\mathrm{IAM} / \mathrm{AP}=$ índice de altura de planta / altura de mazorca; HAMZ = hojas arriba de la mazorca; AFOL = área de la hoja de la mazorca; THOJAS = total de hojas; DTALLO $=$ diámetro de tallo; NRAMA = numero de ramas; LRCENT = longitud de la rama central; CALMAZ = calificación de mazorca; CALPLA = calificación de planta; LMZ = longitud de la mazorca; HILMZ = hileras por mazorca; DMZ = diámetro de mazorca; LGR = longitud de grano; AGR = ancho de grano; GGR = grosor de grano; DOLO $=$ diâmetro de olote; DME $=$ diámetro de medula.

El análisis de conglomerados detectó cinco grupos, con un coeficiente de determinación $\left(\mathrm{R}^{2}\right)$ de 0.4 (Figura 3). Los Grupos I, II, III, IV y V se formaron con 3, 10, 11, 15 y 17 variedades, respectivamente, los cuales se describen a continuación, con base en las 12 variables que contribuyeron más a la variabilidad. El Grupo I, formado por tres materiales mejorados, se caracterizó por ser el más tardío, con los mayores valores de número de: hojas arriba de la mazorca (6), área de la hoja de mazorca $\left(708.1 \mathrm{~cm}^{2}\right)$, longitud de mazorca $(14.7 \mathrm{~cm})$, diámetro de olote $(2.4 \mathrm{~cm})$ y diámetro de médula $(0.7 \mathrm{~cm})$, así como por tener la menor longitud de grano (Cuadro 4). En general, estas plantas fueron de ciclo largo, vigorosas, con mazorca larga y olote grueso.

El Grupo II se formó con 10 poblaciones, de las cuales nueve fueron de la comunidad de San José de Gracia; entre sus características distintivas se encontraron las siguientes: plantas precoces, reducida área de la hoja de la mazorca $\left(565 \mathrm{~cm}^{2}\right)$, mazorcas con el menor diámetro $(4.2$ $\mathrm{cm})$, menor número de hileras (11.3) y mayor longitud $(13.6 \mathrm{~cm})$, con grano más ancho $(9.1 \mathrm{~mm})$ y de mayor grosor $(4.2 \mathrm{~mm})$, de olote delgado $(2 \mathrm{~cm}$ de diámetro) y con diámetro de médula de $0.5 \mathrm{~mm}$. 
El Grupo III estuvo constituido por 10 poblaciones colectadas en comunidades ubicadas al centro-noroeste del municipio: San Andrés (3), Molcaxac (4), San José (1), Santa Cruz (1), San Luis (1) y el híbrido 'H-7583'. Estos genotipos presentaron la mayor área de la hoja de la mazorca entre las poblaciones locales $\left(606 \mathrm{~cm}^{2}\right)$ y valores intermedios de longitud de la mazorca, longitud de grano, ancho de grano y diámetro de olote.

El Grupo IV se formó por 15 materiales de los cuales 11 fueron colectados en la comunidad de Santa Cruz, tres en Molcaxac y uno de San Luis Tehuizotla; este grupo se caracterizó por tener las poblaciones locales más tardías (91 d a floración femenina), con mazorca de $12.6 \mathrm{~cm}$ de largo y mayor número de hileras (14), valores bajos en diámetro de olote y diámetro de médula, con 2.24 y 0.54 $\mathrm{cm}$, respectivamente y con el grano más delgado (8.1 $\mathrm{mm})$.

El Grupo V fue el más numeroso con 17 poblaciones y el más variable en cuanto al origen de sus accesiones, las cuales fueron colectadas en las comunidades del centro sur del municipio: San José de Gracia (5), San Luis Tehuizotla (4), San Andrés Mimihuapan (4), Buenos Aires (2), Santa Cruz (1) y Molcaxac (1); el grupo se caracterizó por ser el más precoz en días a floración femenina $(87 \mathrm{~d})$, de mayor diámetro de mazorca $(4.5 \mathrm{~cm})$, de mayor longitud de grano $(1.4 \mathrm{~cm})$ y menor grosor de grano $(0.4 \mathrm{~cm})$.

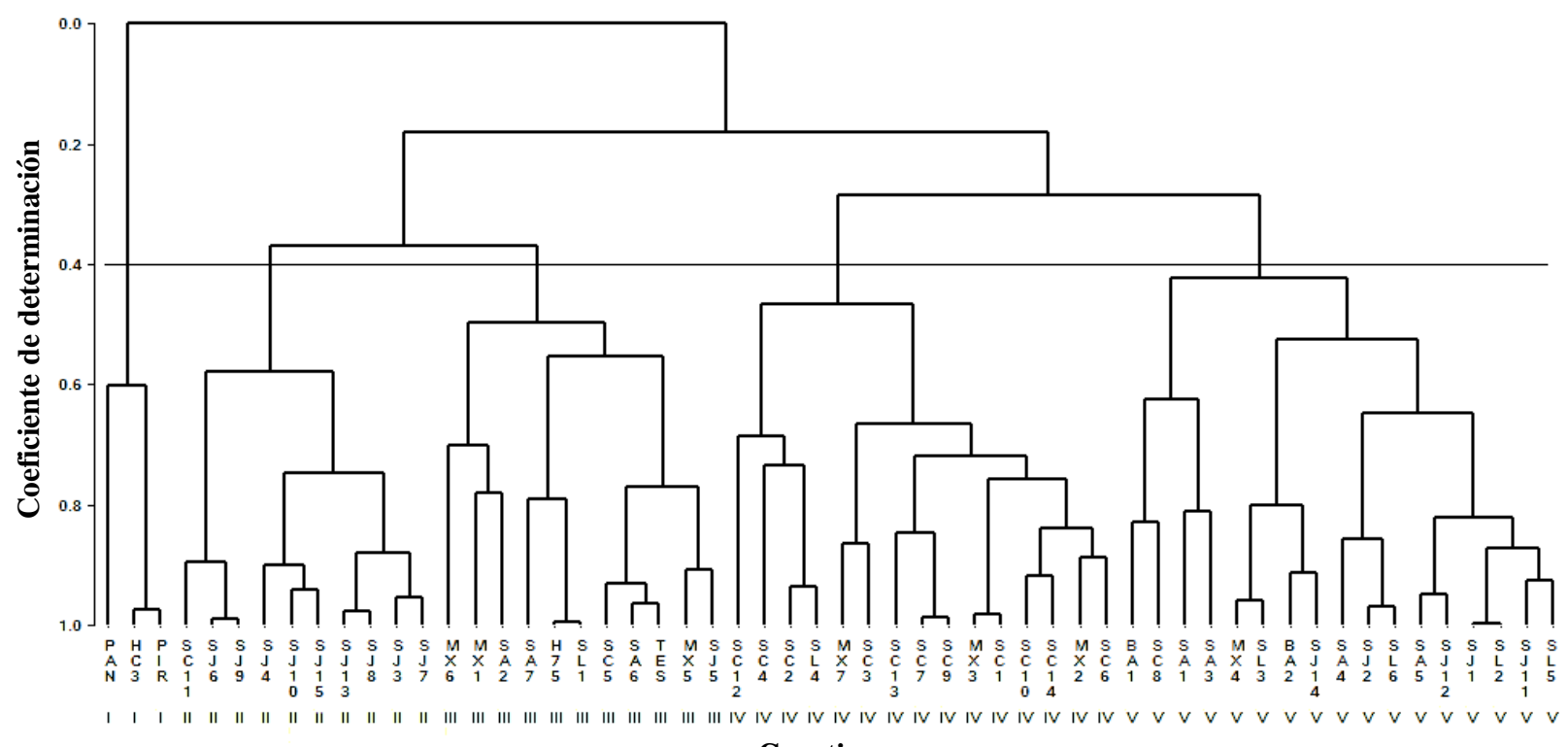

Genotipo

Figura 3. Dendograma de 56 poblaciones de maíz, obtenido con el método de Ward.

Cuadro 4. Promedios por grupo de los cinco obtenidos con el análisis de conglomerados para las 56 variedades de maíz caracterizadas, en el municipio de Molcaxac, Puebla. 2007.

\begin{tabular}{lcccccccccccc}
\hline Grupo & $\begin{array}{c}\text { DFF } \\
(\text { días })\end{array}$ & HAMZ & $\begin{array}{c}\text { AFOL } \\
\left(\mathrm{cm}^{2}\right)\end{array}$ & CALPLA & $\begin{array}{c}\text { LMZ } \\
(\mathrm{cm})\end{array}$ & HILMZ & $\begin{array}{c}\text { DMZ } \\
(\mathrm{cm})\end{array}$ & $\begin{array}{c}\text { LGR } \\
(\mathrm{mm})\end{array}$ & $\begin{array}{c}\text { AGR } \\
(\mathrm{mm})\end{array}$ & $\begin{array}{c}\text { GGR } \\
(\mathrm{mm})\end{array}$ & $\begin{array}{c}\text { DOLO } \\
(\mathrm{cm})\end{array}$ & $\begin{array}{c}\text { DME } \\
(\mathrm{cm})\end{array}$ \\
\hline I & 92 & 6.5 & 708.1 & 2.97 & 14.7 & 13.6 & 4.2 & 11.5 & 8.39 & 3.79 & 2.4 & 0.68 \\
II & 88 & 5.2 & 565.2 & 3.57 & 13.6 & 11.3 & 4.2 & 13.1 & 9.11 & 4.22 & 2.0 & 0.47 \\
III & 89 & 5.3 & 606.5 & 3.36 & 13.6 & 12.5 & 4.4 & 13.3 & 8.74 & 3.83 & 2.2 & 0.54 \\
IV & 91 & 5.3 & 568.6 & 3.31 & 12.6 & 14.4 & 4.4 & 13.3 & 8.12 & 3.90 & 2.2 & 0.56 \\
V & 87 & 5.3 & 566.7 & 3.54 & 12.7 & 13.5 & 4.5 & 14.3 & 8.14 & 3.62 & 2.0 & 0.50 \\
\hline
\end{tabular}

$\mathrm{DFF}$ = dias a floración femenina; HAMZ = hojas arriba de la mazorca; AFOL = área de la hoja de la mazorca; CALPLA = calificación de planta; LMZ = longitud de mazorca; HILMZ = hilera por mazorca; DMZ = diámetro de mazorca; LGR = longitud de grano; AGR = ancho de grano; GGR = grosor de grano; $\mathrm{DOLO}=$ diámetro de olote; DME = diámetro de medula. 
El agrupamiento de las variedades coincidió parcialmente con las localidades donde éstas fueron colectadas, lo cual indica adaptación de las mismas a esas condiciones particulares. El Grupo II estuvo formado principalmente con poblaciones de la comunidad de San José de Gracia, mientras que el Grupo III aglutinó poblaciones de las comunidades del centro-noroeste, y el Grupo IV con poblaciones de comunidades ubicadas en el centro-sur del municipio. Estos agrupamientos también están influenciados por el gradiente de variación altitudinal de norte a sur que se presenta en el área de estudio, pues la comunidad de Molcaxac está ubicada entre 1800 y $2000 \mathrm{msnm}$, con un clima semicálido (temperatura media anual de $17{ }^{\circ} \mathrm{C}$ ) y San José de Gracia a $2200 \mathrm{msnm}$, con clima templado (temperatura media anual de $9{ }^{\circ} \mathrm{C}$ ). Los resultados concuerdan con lo reportado por López et al. (2005) y González et al. (2006), quienes estudiaron la diversidad del maíz Zapalote Chico en el Istmo de Tehuantepec y del Cacahuacintle en el Valle de Toluca, respectivamente, donde también observaron influencia de gradientes altitudinales.

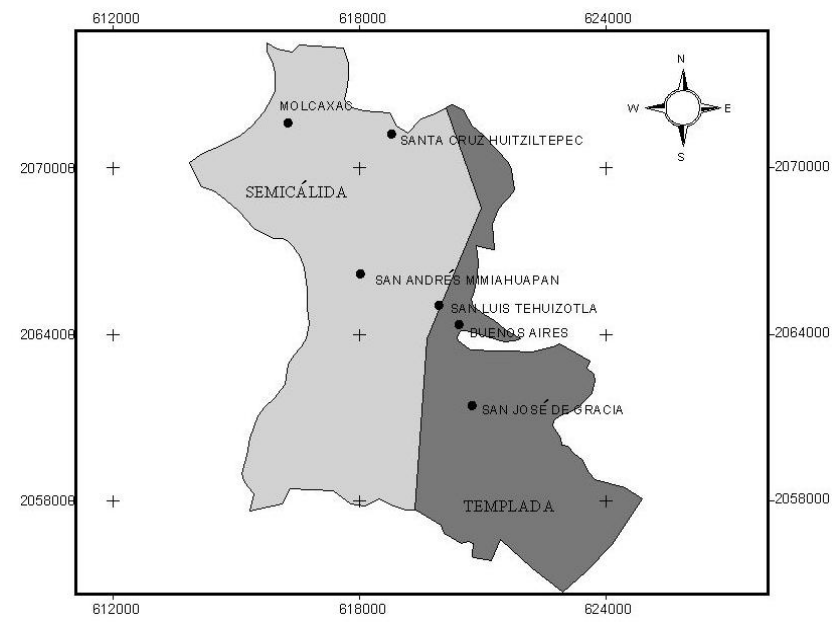

Figura 4. Ubicación de las comunidades de colecta de accesiones en el municipio de Molcaxac.

Las únicas localidades que cuentan con riego son Santa Cruz Huitziltepec y San Luis Tehuizotla, lo que también explica el agrupamiento de las poblaciones de acuerdo con la localidad de colecta (Figura 4). El Grupo II está adaptado a un ciclo de producción más corto y a condiciones más severas de escasez de lluvia, mientras que el Grupo III se integra con genotipos que combinan características de adaptación a las dos condiciones ambientales predominantes del municipio, ya que las comunidades de origen de las poblaciones de este grupo se ubican en una zona intermedia entre dichas condiciones. El Grupo IV incluye a variedades desarrolladas en condiciones especiales dentro del municipio, ya que la comunidad de Santa Cruz cuenta con riego, por lo que el ciclo de crecimiento se puede extender y así generar plantas tardías y de mayor vigor. El Grupo V concentró a las accesiones de las comunidades del sur del municipio, donde hay la mayor altitud $(2200 \mathrm{~m})$, clima templado y con el ciclo de producción más restrictivo, lo que ocasiona que las poblaciones de estas localidades tengan una estación de crecimiento más corta. La falta de coincidencia completa de los grupos formados con las comunidades de colecta puede tener su explicación en el hecho de que los agricultores comúnmente intercambian semilla (González et al. 2006).

\section{CONCLUSIONES}

En el municipio de Molcaxac, Puebla, México, existe amplia diversidad genética entre las poblaciones locales de maíz, al grado que algunas de ellas superan o igualan en rendimiento de grano a híbridos comerciales recomendados para la región. Las poblaciones locales MX-6, SA-2 y SC-3 sobresalieron en rendimiento con $5.6,4.8$ y $4.5 \mathrm{t} \mathrm{ha}^{-1}$, respectivamente, en tanto que el mejor hibrido comercial ('H-C-3311') rindió 4.2 t ha' .

Con respecto a los días a floración femenina, las variedades se agruparon en tres niveles de precocidad: precoz, intermedio y tardío, en una proporción de 30, 52 y $18 \%$, respectivamente. El mayor rendimiento se presentó en el estrato de floración precoz, mientras que las variedades tardías fueron las menos rendidoras.

Las variedades nativas también presentaron diversidad genética en color del grano; el orden de abundancia por color es: blanco $>$ azul $>$ amarillo. El maíz blanco cubrió todos los estratos de precocidad, con predominio (52 \%) del estrato intermedio. El maíz azul fue esencialmente intermedio, y la única colecta amarilla fue precoz.

Se identificaron cinco grupos de variedades que difirieron en características fenológicas, morfológicas y agronómicas, que conservan una relación PARCIAL con el origen geográfico de las colectas.

\section{BIBLIOGRAFÍA}

Aceves R E, A Turrent F, J I Cortes F, V Volke H (2002) Comportamiento agronómico del híbrido H-137 y materiales criollos de maíz en el Valle de Puebla. Rev. Fitotec. Mex. 25:339-347.

Barrales D S, A Muñoz O, D Sotres $R$ (1984) Relaciones termopluviométricas en familias de maíz bajo condiciones de temporal. Agrociencia 58:127-139.

Castillo P T, A Muñoz O, A Santos G (1987) Variedades de maíz y frijol sobresalientes en la Mixteca Guerrerense. In: Memoria del Seminario como Aumentar la Producción Agropecuaria y Forestal en la Región Mixteca Oaxaqueña. A Muñoz O, B. 
Dimas C. (comps). Tomo II. SARH, Gobierno del Estado de Oaxaca y Colegio de Posgraduados. Tiltepec, Oaxaca, México. pp:575-581.

Centro de Investigaciones Agrarias (1980) El Cultivo de Maíz en México. Talleres de Industria Gráfica Editorial Mexicana. México, D. F. 148 p.

Cervantes S T, H Mejía A (1984) Maíces nativos del área del Plan Puebla: recolección de plasma germinal y evaluación del grupo tardío. Rev Chapingo 9:64-71.

Cochran W G, G M Cox (1965) Diseños Experimentales. Ed. Trillas. México, D. F. 666 p.

Estrada G J A, C López C, A Muñoz O, H Hernández S (1987) Mejoramiento de la resistencia a sequía en Chiautla, Pue. I. Prueba de variedades de maíz y primer ciclo de selección. In: Memoria del Seminario como Aumentar la Producción Agropecuaria y Forestal en la Región Mixteca Oaxaqueña. A Muñoz O, B Dimas C (comps). Tomo II. SARH, Gobierno del Estado de Oaxaca y Colegio de Posgraduados. Tiltepec, Oaxaca, México. pp:457-468.

García E (1973) Modificaciones al Sistema de Clasificación Climática de Köppen. Instituto de Geografía. UNAM. México, D. F. 246 p.

Gil M A, P A López, A Muñoz O, H López S (2004) Variedades criollas de maíz (Zea mays L.) en el Estado de Puebla, México: diversidad y utilización. In: Manejo de la Diversidad de los Cultivos en los Agroecosistemas Tradicionales. J L ChávezServia, J Tuxill y D I Jarvis (eds). Instituto Internacional de Recursos Fitogenéticos. Cali, Colombia. pp:18-25.

González H A, J Sahagún C, D de J Pérez L, A Domínguez L, R Serrato C, V Landeros F, E Dorantes C (2006) Diversidad fenotípica del maíz Cacahuacintle en el Valle de Toluca, México. Rev. Fitotec. Mex. 29:255-261.

Herrera C B E, F Castillo G, J J Sánchez G, R Ortega P, M M Goodman (2000) Caracteres morfológicos para valorar la diversidad entre poblaciones de maíz en una región: caso la raza Chalqueño. Rev. Fitotec. Mex. 23:335-354.

Herrera C B E, F Castillo G, J J Sánchez G, J M Hernández C, R A Ortega P, M M Goodman (2004) Diversidad del maíz Chalqueño. Agrociencia 38:191-206.

Hortelano S R R, A Gil M, A Santacruz V, S Miranda C, L Córdova $\mathbf{T}$ (2008) Diversidad morfológica de maíces nativos en el Valle de Puebla. Agric. Téc. Méx. 34:189-200.
IBPGR, International Board for Plant Genetic Resources (1991) Descriptors for Maize. International Maize and Wheat Improvement Center-International Board for Plant Genetic Resources. Rome. 29 p.

INEGI, Instituto Nacional de Estadística y Geografía (2004) Anuario Estadístico de Puebla. Tomo II. Instituto Nacional de Estadística, Geografía e Informática. Aguascalientes, Ags. 1004 p.

INEGI, Instituto Nacional de Estadística y Geografía (2007) Anuario Estadístico de Puebla. Tomo II. INEGI; Aguascalientes, Ags. $1284 \mathrm{p}$.

INEGI, Instituto Nacional de Estadística y Geografía (2010) Anuario de Estadística por Entidad Federativa. INEGI-Aguascalientes, Ags. México. 594 p.

Kato Y T A, C Mapes S, L M Mera O, J L Serratos H, R A Bye B (2009) Origen y Diversificación del Maíz: Una Revisión Analítica. Universidad Nacional Autónoma de México, Comisión Nacional para el Conocimiento y Uso de la Biodiversidad. México, D. F. 116 p.

López H A de J, A Muñoz O (1984) Relación de la coloración del grano con la precocidad y la producción en maíces de Valles Altos. Rev. Chapingo 9:31-37.

López R G, A Santacruz V, A Muñoz O, F Castillo G, L Córdova T, H Vaquera $H$ (2005) Caracterización morfológica de poblaciones nativas de maíz del istmo de Tehuantepec, México. Interciencia 30:284-290.

Mohammadi S A, B M Prasanna (2003) Analysis of genetic diversity in crop plants-salient statistical tools and considerations. Crop Sci. 43:1235-1248.

Muñoz O A (2005) Centli Maíz. Ed. América. 2da ed. México, D. F. $210 \mathrm{p}$.

Ortega P R (2003) La diversidad del maíz en México. In: Sin Maíz no Hay País. G Esteva, C Marielle (coords). Consejo Nacional para la Cultura y las Artes, Dirección General de Culturas Populares e Indígenas. México, D. F. pp:123-154.

SAS Institute (2004) SAS/STAT 9.0. User's Guide. SAS Institute Inc. Cary, NC. pp:1731-1900.

Serratos H J A (2009) El Origen y la Diversidad del Maíz en el Continente Americano. Greenpeace. Ciudad de México, México. 33 p. 